\title{
TEMPERATURE ANISOTROPY IN THE PRESENCE OF ULTRA LOW FREQUENCY WAVES IN THE TERRESTRIAL FORESHOCK
}

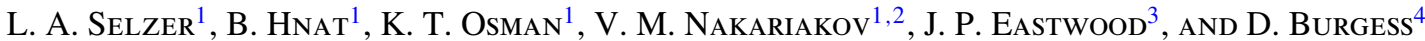 \\ ${ }^{1}$ Centre for Fusion, Space and Astrophysics, University of Warwick, Coventry CV4 7AL, UK; L.A.Selzer@warwick.ac.uk \\ ${ }^{2}$ Central Astronomical Observatory at Pulkovo of the Russian Academy of Sciences, 196140 St. Petersburg, Russia \\ ${ }^{3}$ Space and Atmospheric Physics, The Blackett Laboratory, Imperial College London, London, UK \\ ${ }^{4}$ School of Physics and Astronomy, Queen Mary University of London, UK \\ Received 2013 July 15; accepted 2014 April 15; published 2014 May 21
}

\begin{abstract}
We report the first study of the correlation between elevated solar wind core plasma temperatures and temperature anisotropy in the terrestrial foreshock. Plasma temperature is enhanced near the fire hose marginal stability threshold in the presence of ultra low frequency (ULF) large amplitude magnetic perturbations, which are intrinsically righthand circularly polarized. Direct comparison of contemporaneous anisotropic temperatures in the upstream solar wind and the foreshock suggests that the net heating of plasma is mediated via increase of the parallel temperature in the foreshock region where the ULF waves are present. We consider the possibility that a mechanism based on Landau damping, where solar wind plasma temperature parallel to the background magnetic field is increased by interaction with oblique compressible fast magneto-acoustic ULF waves, influences temperature anisotropy.
\end{abstract}

Key words: plasmas - polarization - solar-terrestrial relations - solar wind - Sun: oscillations - waves

Online-only material: color figures

\section{INTRODUCTION}

The terrestrial foreshock is an ideal laboratory for the in situ study of the interaction between a quasi-stationary shock and a collisionless plasma. In particular, the region upstream of the quasi-parallel shock supports a plethora of dispersive waves, which can interact with particles in both a resonant and non-resonant manner. These interactions may lead to particle acceleration and plasma heating. An understanding of heating mechanisms in collisionless quasi-parallel shocks is crucially important to many outstanding space plasma problems, such as coronal heating and the non-adiabatic expansion of the solar wind. This work presents observational evidence for foreshock dissipation mediated by the fire hose instability.

Early foreshock studies identified multiple wave generation mechanisms for both left- and right-hand circularly polarized waves in the plasma frame (Heppner et al. 1967; Fairfield et al. 1971; Russell et al. 1971; Barnes 1970). The beam instability is the primary mechanism producing right-hand polarized transverse waves, propagating parallel and anti-parallel to the interplanetary magnetic field. Waves traveling along the beam are resonant with the ion beam population, while waves propagating anti-parallel to the beam may become unstable to the non-resonant fire hose instability in the presence of temperature anisotropy (Sentman et al. 1981; Gary et al. 1998). The plasma becomes unstable to fire hose when $P_{\|}-P_{\perp}>B^{2} / 2 \mu_{0}$, i.e., the plasma pressure parallel to the magnetic field direction is greater than that perpendicular to the magnetic field direction by an amount exceeding the local magnetic pressure.

The linear phase of the beam instability is well understood: long wavelength transverse electromagnetic fluctuations are produced and these scatter and isotropize the beam particles, reducing the source of the instability. The interaction of these waves with the unstable population may, however, be diminished due to advection of waves by the solar wind as well as their refraction (Scholer et al. 2003). The non-linear phase of this process is less understood, but it is known that the generated fluctuations can interact with the bulk ion population and affect global plasma properties. Two general mechanisms may then be considered. If an isolated mode becomes unstable and the nonlinear temporal scale exceeds the linear one, coherent waves may lead to the wave particle interactions. If the broadband spectrum is generated and nonlinearity is strong, the energy may cascade to large scales, modifying plasma parameters in order to suppress the instability (Rosin et al. 2011; Quest \& Shapiro 1996). Here, we consider the hypothesis that, in the nonlinear phase of the beam instability, the ultra low frequency (ULF) waves influence the temperature anisotropy of the core ion population, which may lead to a "secondary instability" of the fire hose or proton cyclotron (PC) type.

We present the analysis of core proton temperature anisotropy associated with intrinsically left- and right-hand polarized ULF waves in the terrestrial foreshock. Our results suggest an ordering: the proximity of the core plasma distribution to the fire hose marginal stability threshold coincides with the presence of right-hand polarized ULF waves, while the left-hand polarized waves coincide with the proximity to PC and/or mirror mode instability threshold. Plasma temperature is elevated at both instability thresholds and we examine if this increase can be associated with the net heating of core ions. We will comment on two distinct mechanisms that can lead to marginal stability of the plasma: large magnetic field strength fluctuation and particle scattering by obliquely propagating, compressible ULF waves. While being of broad interest to the terrestrial foreshock community, these findings may have important implications for solar wind studies. Elevated plasma temperatures have recently been observed near kinetic instability thresholds (Maruca et al. 2011) and have also been associated with large amplitude magnetic fluctuations (Osman et al. 2012).

\section{DATA}

We used Cluster high-resolution $(\approx 22 \mathrm{~Hz})$ magnetic field data from the Fluxgate Magnetometer (FGM) instrument (Balogh et al. 1997) and spin (4 s) cadence Cluster Ion SpectrometerHot Iron Analyser (CIS-HIA) onboard-calculated solar wind moments which measure core population properties (Réme 
Table 1

Cluster Intervals Studied in the Letter

\begin{tabular}{lcllcc}
\hline \hline Date & Times & \multicolumn{1}{c}{ S/c } & \multicolumn{1}{c}{ B Field } & S/c Polarization & Wave Vector (B Aligned Coord.), \\
\hline 2004 Jan 27 & $03: 50-04: 35$ & C1 & Sunward & Left-hand & $(0.99,0.16,-0.04)$ \\
2002 Feb 16 & 06:19-06:29 & C1, C3 & Anti-sunward & Left-hand & $(-0.81,0.58,0.74)$ \\
2002 Feb 20 & $22: 00-22: 06$ & C1 C3 & Sunward & Left-hand & $(0.83,0.55,-0.01)$ \\
2010 Feb 19 & $00: 16-01: 10$ & C1 & Anti-Sunward & Left-hand & $(-0.84,-0.29,-0.45)$ \\
2002 Feb 21 & $22: 19-22: 29$ & C1, C3 & Sunward & Left-hand & $(-0.98,-0.14,-0.14)$ \\
2002 Feb 11 & $22: 04-22: 14$ & C1, C3 & Anti-sunward & Left-hand & $(0.86,-0.39,-0.31)$ \\
2002 Mar 27 & $07: 15-07: 25$ & C1, C3 & Anti-sunward & Left-hand & $(0.86,-0.51,0.03)$
\end{tabular}

et al. 1997). All HIA samples were collected in mode 5, which measures the core population of the solar wind beam. In principle, a small fraction of a field-aligned beam may contribute to these measurements, increasing the parallel temperature. Here, we assume that such effects would lead to small variations in the onboard computed moments, but would not result in large and systematic changes reported here for some of the intervals. Also, the HIA instrument cannot differentiate between different ion species, thus the effects of $\alpha$ particles are folded into these measurements. The field of view of the instrument in this mode is approximately $\pm 22^{\circ}$. Visual inspection of the velocity distributions, using the CLWeb (http://clweb.cesr.fr) ground computed product, confirmed that this field of view is sufficient to capture the majority of the distribution functions for selected intervals. For a core proton velocity distribution, a single bi-Maxwellian has been found to be a good approximation (Kasper et al. 2002; Hellinger et al. 2006). All intervals had core plasma thermal velocities much below that of the solar wind.

The data set consists of four intervals of intrinsically righthand polarized waves and three intervals containing intrinsically left-hand polarized waves; these intervals are listed in Table 1. When available, measurements from spacecraft $C 1$ and $C 3$ were treated as independent measurements. Separation between these spacecraft was $\approx 10,000 \mathrm{~km}$, except for interval 4 , when spacecraft $C 1$ and $C 3$ were considerably closer. The plasma frame (intrinsic) polarization will be used to distinguish between types of wave intervals-we will use abbreviations IR for intrinsically right-handed and IL for intrinsically left-handed intervals.

In order to compare foreshock anisotropy with that of the ambient solar wind upstream, we have identified one interval of WIND spacecraft data for which the time shifted magnetic field vector of WIND has been correlated with that observed by Cluster. This is the Cluster interval of 2010 February 19 spanning foreshock times 00:16-01:10. We examined WIND Solar Wind Experiment (SWE) ion anisotropy data with time cadence of $92 \mathrm{~s}$. A time shift of 3200 was obtained from cross correlation of WIND (3 s cadence) and Cluster (4 s cadence) magnetic field data.

Figure 1 presents summary plot for the canonical intervals: 2002 February 11 for the left- and 2004 January 27 for the righthand polarization. In all panels blue crosses correspond to the IL interval and red circles to the IR interval. Panels (a) and (b) show the maximum variance transverse field component of the magnetic field for the IR and IL intervals, respectively, and these show clear large amplitude fluctuations with the period of about $30 \mathrm{~s}$. These fluctuating components are calculated by rotating the magnetic field into a field-aligned coordinate system given by $\hat{e}_{1}=\hat{\mathbf{b}}, \hat{e}_{\perp 1}=\mathbf{B}-(\mathbf{B} \cdot \hat{\mathbf{b}}) \hat{\mathbf{b}}$ and $\hat{e}_{\perp 2}=\left(\hat{e}_{1} \times \hat{e}_{\perp 1}\right)$ unit vectors, where $\hat{\mathbf{b}}=\mathbf{B} / B$. Panel (c) shows the power spectra of components shown in panels (a) and (b). Two vertical lines
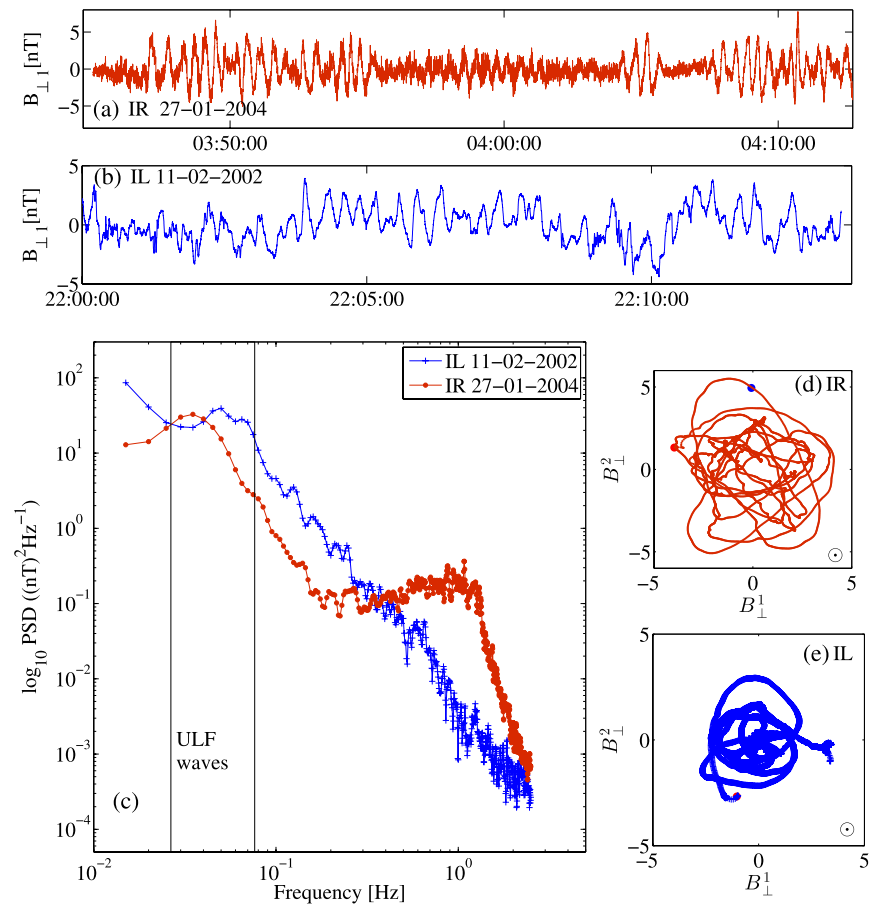

Figure 1. Selected time traces of the maximum variance transverse magnetic field component $B_{\perp 2}$ for the canonical intervals of (a) right (2004 January 27 03:46-04:15) and (b) left-hand polarized (2002 February 11 22:00-22:08) waves. Panel (c): power spectra of the complete data (times in Table 1 for canonical intervals of right- (red circles) and left-hand (blue crosses) polarized waves. Panels (d) and (e) show spacecraft polarization of right-hand polarized waves and left-hand polarized waves, respectively.

(A color version of this figure is available in the online journal.)

mark the spectral peaks of the ULF waves, with the range of periods between $\approx 20-50 \mathrm{~s}$ (frequency $v \approx 0.02-0.05 \mathrm{~Hz}$ ). Hodograms for the IL and IR intervals, with the magnetic field direction out of page, are shown in panels (d) and (e) of Figure 1. These show circular polarization and were calculated for 3-5 periods of the ULF wave in the part of the interval where the waves activity is clear. The ULF waves were identified as fast magnetoacoustic waves based on their oblique wave numbers, sense of polarization, and the correlation of magnetic strength and plasma density fluctuations (not shown here). The IR power spectra also has a peak around $1 \mathrm{~Hz}$, and this feature may be related to the "1 $\mathrm{Hz}$ whistlers" associated with electron foreshock (Hoppe et al. 1982; Sentman et al. 1983; Burgess 1997). The study of these waves is outside the scope of this work, but it should be mentioned that they can also interact with the core of the ion distribution.

In order to determine the intrinsic plasma frame polarization of the ULF waves, a multi-spacecraft delay analysis (Paschmann 

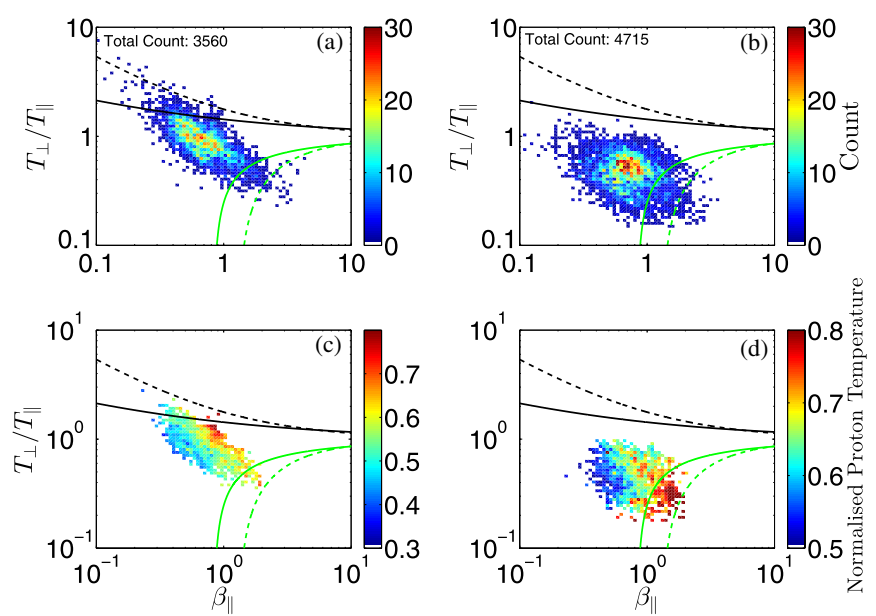

Figure 2. (a) Ensemble-averaged distribution of counts for all intervals with lefthand polarization. (b) Same as (a) for the ensemble of right-hand polarization. (c) Pixelated plot of the ensemble-averaged, normalized proton temperature at $\left(\beta_{\|}, T_{\perp} / T_{\|}\right)$for the left-handed intervals. Only bins with counts larger than three are considered. (d) Same as (c) for the right-hand intervals. The curves show the maximum growth rates for proton cyclotron instability (solid black curve), mirror instability (dashed black curve), oblique fire hose instability (green dashed curve), and the parallel fire hose instability (solid green curve). The growth rates for $\gamma_{\max }=10^{-3} w_{\mathrm{ci}}$ are plotted.

(A color version of this figure is available in the online journal.)

\& Daly 1998) was applied. Essentially, cross correlation between spacecraft provides time of travel information, while the autocorrelation of each signal gives its frequency. An optimization procedure is then used to obtain the direction of the wave vector. Table 1 lists both the plasma frame polarization and the wave propagation direction following subtraction of the solar wind flow speed. The wave vectors (not normalized to unity) are represented in a magnetic-field-aligned coordinate system, where the $x$-axis is along the mean magnetic field direction, the $z$-axis is perpendicular to both the velocity and magnetic fields, and the $y$-axis completes the right-handed coordinate system. We note that in the case of interval 4 in the table, spacecraft configuration made the wave vector estimation very difficult and may include large error. However, the intrinsic right-hand polarization is also supported by the fact that the magnetic field strength and density perturbations were correlated.

\section{RESULTS}

A parameter space given by proton temperature anisotropy $R=T_{\perp} / T_{\|}$and parallel plasma beta $\beta_{\|}=2 \mu_{0} n k_{B} T_{\|} / B^{2}$ (Hellinger et al. 2006; Bale et al. 2009; Osman et al. 2012) is used to visualize a possible link between temperature anisotropy and core proton temperatures. Cluster HIA temperature data has been smoothed over a window of four neighboring points in order to construct these quantities. Figure 2, panels (a) and (b) shows the distributions of counts for the left- and right-hand wave intervals, respectively. The ensemble-averaged distribution of temperatures, based on core ion distributions for intervals of specific polarization, are shown in panels (c) and (d) of Figure 2. Only the temperature in bins with more than five counts was considered for these plots. The theoretical marginal stability thresholds (Hellinger et al. 2006; Marsch et al. 2004) of the PC instability (solid black curve), mirror instability (dashed black curve), and oblique (dashed green curve) and parallel (solid green curve) fire hose instabilities are shown in all panels. The position of these instability threshold lines is
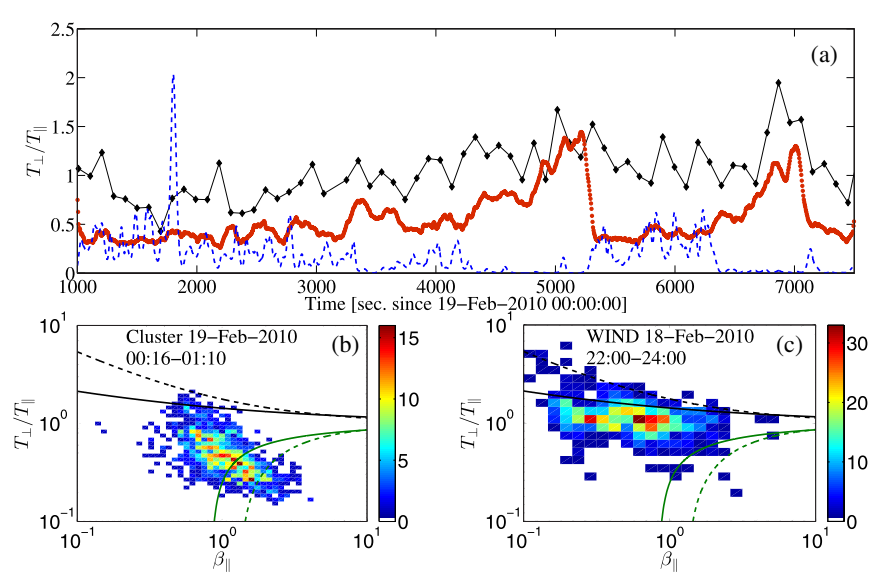

Figure 3. (a) Comparison of the upstream solar wind anisotropy, as seen by the WIND spacecraft (black diamonds) with that observed by the Cluster spacecraft (red solid line). The blue curve shows the integrated wavelet coefficient power in the ULF frequencies of $0.02-0.06 \mathrm{~Hz}$. (b) and (c) Distribution of counts for the Cluster and WIND spacecraft.

(A color version of this figure is available in the online journal.)

somewhat arbitrary, since the exact position requires the ratio of growth rate and ion cyclotron frequency, $\gamma / \omega_{\mathrm{ci}}=0$. In practice this limit is approximated, similarly to other studies, by a small value $\gamma / \omega_{\mathrm{ci}}=10^{-3}$. We note that the axes of these plots are related via their common dependence on the parallel temperature.

The data plotted in the $\left(\beta_{\|}, R\right)$ plane falls into two distinct categories depending upon the intrinsic ULF wave polarization. The count distribution for the IR intervals peaks close to the marginal stability line of the fire hose instability. There is also a strong enhancement in temperature near this region. The temperature for the points located near the fire hose marginal stability curves is usually factor of two to three higher compared to those located near the $R=1$ line. The left-hand intervals show similar elevated temperatures near the PC/mirror instability thresholds. While the differences between the PC and the mirror mode instability threshold are small for $\beta_{\|}>1$, the mirror mode is linearly polarized and thus can be excluded from our consideration. Note that for the IL intervals, the count distribution has its maximum nearer to the isotropy line and the outliers stretch from the PC to fire hose marginal stability lines. This, and a smaller number of samples contributing to the plot for the left-hand polarized waves, makes the interpretation difficult.

Elevated temperatures around the marginal thresholds of the kinetic instabilities do not imply net heating of plasma. Indeed, available energy may simply be transferred between the perpendicular and parallel particle motions. In addition, similar correlations between the ion temperature and the anisotropy have been previously reported for the ambient solar wind (Bale et al. 2009; Osman et al. 2012). Thus, it is crucial to verify that the upstream solar wind conditions are not fully responsible for these observations. Such a test requires a contemporaneous observation of upstream and foreshock plasmas. We have identified one interval (interval 4 of Table 1) where the WIND and Cluster spacecraft magnetic fields correlated well after a forward time shift of $3200 \mathrm{~s}$, applied to WIND data.

Panel (a) of Figure 3 shows WIND (black diamonds) and Cluster (red circles) measured temperature anisotropy, with WIND data shifted forward in time by $3200 \mathrm{~s}$. The blue solid line corresponds to the integrated wavelet power for the ULF frequencies in the range $0.02-0.06 \mathrm{~Hz}$. While the upstream 

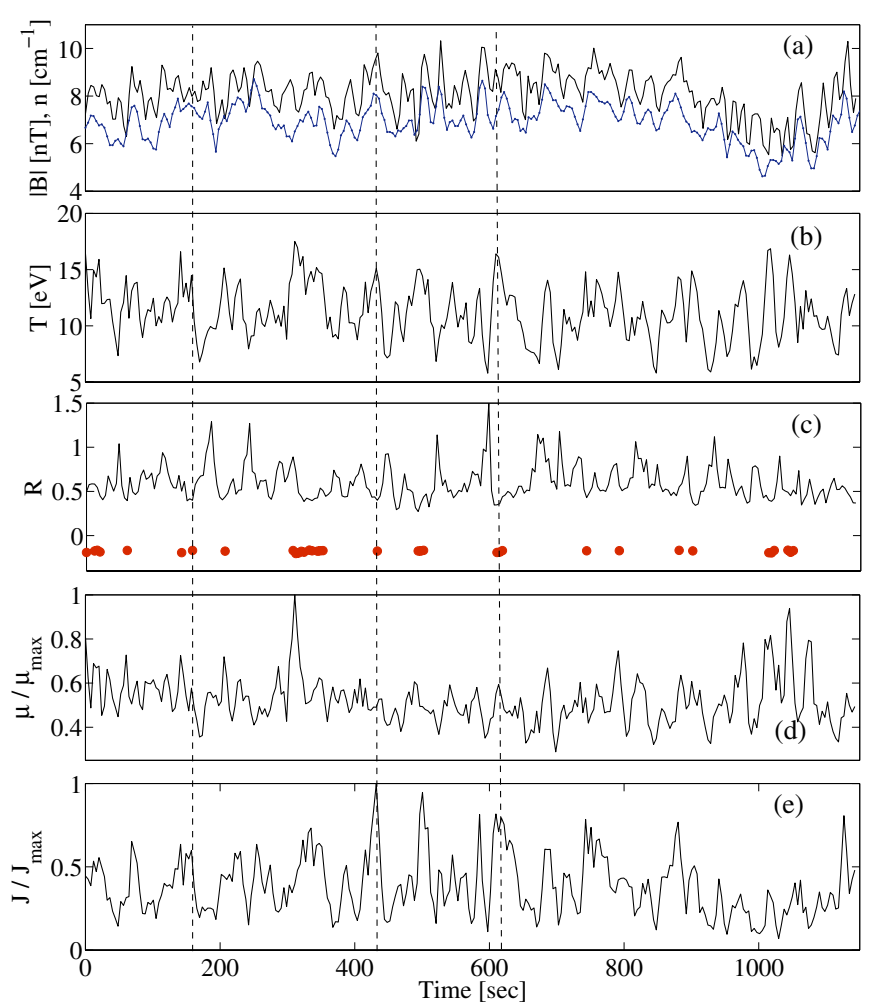

Figure 4. (a) Magnetic field strength downsampled to $4 \mathrm{~s}$ resolution and HIA proton density, (b) ion temperature, temperature anisotropy ratio, (c) $R$, (d) normalized first adiabatic invariant, and (e) normalized second adiabatic invariant for the interval 2004 January 27 4:07-04:26. Solid symbols (circles) in panel (c) indicate the temporal position of the 30 highest temperature measurements. Dashed vertical lines indicate examples of drops in the anisotropy trace, which do not coincide with significant magnetic field strength change.

(A color version of this figure is available in the online journal.)

solar wind conditions broadly modulate foreshock conditions, it is clear that the temperature anisotropy in the foreshock is much lower, as compared with WIND. We find a sharp increase in the mean (and median) parallel temperature, from $27 \mathrm{eV}$ to about $76 \mathrm{eV}$ (factor of $\approx 3$ ), between the upstream and the foreshock plasma. The perpendicular temperature shows only a small increase (within the error bar). Interestingly, the power in the ULF waves appear to correlate well with the observed anisotropy-where the ULF wave activity exhibits a sharp decrease, the anisotropy rises and reaches values observed by WIND in the ambient solar wind.

We have also examined intervals $1-4$, listed in Table 1 , for times when Cluster's orbit appeared to be in the ambient solar wind. While this is not equivalent to the upstream-foreshock analysis, we have identified similar behavior in interval 3, with the parallel temperature increase by a factor of $\sim 2$ between the solar wind and the foreshock part of the Cluster trajectory.

We next examine the temporal dynamics of the measurements, presented in Figure 2(b), on the $\left(\beta_{\|}, R\right)$ plane. The aim is two-fold: to establish if points contributing to the temperature peak come from a single time interval within the time series and to check if changes in anisotropy are always associated with changes in magnetic field strength. In order to facilitate such a test, we examine the observed correlation between temperature and anisotropy, $R$, for a time interval where $\beta_{\|}$does not vary significantly. We also consider the relationship between anisotropy and the macroscopic versions of adiabatic invariants.

Figure 4 shows the quantity of interest for each data point in the interval 2004 January 27 between times 04:05-04:25. Panel (a) is a trace of the magnetic field magnitude downsampled to $4 \mathrm{~s}$ resolution of the plasma data as well as proton density perturbations. It is clear that the density is well correlated with magnetic field strength perturbations. Panel (b) shows the temperature for this time period and panel (c) shows the temperature anisotropy, $R$. Solid filled circles in panel (c) represent the temporal position of the 30 highest temperature measurements. Panels (d) and (e) show the temporal traces of the first $\left(\mu=p_{\perp} / 2 B\right)$ and second (approximated by $J=p_{\|} B^{2}$ ) adiabatic invariants normalized to their maximum values, respectively. In the fluid description these adiabatic invariants should be constant, but we observe significant fluctuations on small timescales, which are of order $\approx 21 \%$ for $\mu$ and $\approx 46 \%$ for $J$.

It is clear that the points close to the fire hose marginal stability line $(R \leqslant 0.5)$ do not originate from a single cluster of data. We observe an intermittent behavior where the temperature anisotropy varies considerably and does not follow periodic oscillations of the magnetic field components. The highest temperatures coincide with points of minimum anisotropy and these are also evenly distributed throughout this interval. We note that between times $t_{0}=900 \mathrm{~s}$ and $t_{1}=1150 \mathrm{~s}$, the magnetic field magnitude and the anisotropy both decrease, but this does not coincide with a significant increase of concentration for temperature enhancements. Close examination of the traces in Figure 4 shows that sharp drops in the anisotropy trace do not show direct correlation with magnetic field strength, that is, they do not coincide with magnetic field strength maxima or minima exclusively. At the same time, however, the times of elevated temperatures coincide with large deviations in the second adiabatic invariant, suggestive of parallel heating via wave particle interactions.

\section{DISCUSSION AND CONCLUSIONS}

Results presented above indicate a correlation among higher plasma temperature, the presence of the right-hand polarized waves, and plasma proximity to the fire hose marginal stability threshold. Similar correlation is observed for the left-hand polarized waves, close to the marginal stability line of the PC instability. Correlations are not synonymous with causality and, given the complexity of plasma dynamics in the foreshock region, the interpretations of these results are difficult.

The proximity of the core plasma distribution to the fire hose marginal stability line does not imply that the instability itself must necessarily control the dynamics of the plasma. By monitoring the upstream conditions, using WIND spacecraft, we have demonstrated that the temperature anisotropy is modified in the foreshock in such a way that $T_{\perp} \ll T_{\|}$. The enhancement in the total temperature correlates well with the factor of two to three increase in $T_{\|}$observed in two intervals. The results suggest that the power in ULF waves is also strongly correlated with the observed anisotropy in the foreshock, at least for the right-hand polarized waves.

One possible interpretation of these results is to suggest that the "secondary" fire hose instability is indirectly driven by the right-hand polarized ULF waves. Aided with observations presented in Figure 4, we consider two distinct mechanisms that can modify plasma temperature anisotropy in the presence of large magnetic field fluctuations. If the first adiabatic invariant is conserved, any decrease in the magnetic field strength will lead to an equivalent decrease in the perpendicular pressure, $p_{\perp}$, pushing the bulk plasma fluctuations toward the fire hose unstable region. Our results, however, show that the anisotropy changes are not exclusively associated with such magnetic field 
decreases. This suggests a second mechanism in the form of Landau damping of oblique fast magnetosonic ULF waves, which has often been discussed in the context of cosmic ray acceleration (see, e.g., Schlickeiser \& Miller 1998, and references therein). The wave energy is transferred into parallel particle motion in the presence of a compressive magnetic field component. Compressibility is essential for the viability of this proposed heating mechanism and it is clearly visible from panel (a) of Figure 4 that these right-hand polarized waves do perturb the magnetic field magnitude. For large amplitude waves, particles with super-Alfvénic velocities will experience a large mirror force $F_{m}=-\left(m v_{\perp}^{2} / 2 B\right) \nabla_{\|} B$ and will be accelerated if they experience head-on reflection. The net heating is a result of the difference between the occurrence of wave-particle interactions for particles moving with (lower occurrence) and against (higher occurrence) the wave.

In summary, the results presented here pertain directly to a correlation between elevated temperatures and temperature anisotropy in the terrestrial foreshock. However, these could also be relevant to studies of the solar wind plasma, where similar correlations have also been observed. For example, given a small number of points, which populate regions close to marginal stability thresholds in the solar wind studies (Bale et al. 2009; Kasper et al. 2002), it is reasonable to suggest that these signatures may arise from wave activity near relatively small number of coherent structures, such as shocks (Wilson III et al. 2009). The Landau damping of ULF waves, identified as a possible driving mechanism for the fire hose instability, may also be important in solar wind turbulence, where oblique fast magneto-acoustic waves of low frequency are believed to be present, for example, in the foreshocks of quasi-parallel coronal mass ejection shocks (Gonzalez-Esparza et al. 1996; Tu et al. 1989).
This work was supported by the UK STFC and EU Marie Curie "Turboplasmas" funding. D.B. acknowledges support of the STFC grant ST/J001546/1. J.E. acknowledges support of the STFC grant ST/G00725X/1.

\section{REFERENCES}

Bale, S. D., Kasper, J. C., Howes, G. G., et al. 2009, PRL, 103, 211101

Balogh, A., Dunlop, M. W., Cowley, S., et al. 1997, SSRv, 79, 65

Barnes, A. 1970, CosEl, 1, 90

Burgess, D. 1997, AdSpR, 20, 673

Fairfield, D. H. 1971, JGR, 74, 3541

Gary, S. P., Li, H., O’Rourke, S., \& Winske, D. 1998, JGR, 103, 14567

Gonzalez-Esparza, J. A., Smith, E. J., Balogh, A., \& Phillips, J. L. 1996, A\&A, 316,323

Hellinger, P., Trávníček, P., Kasper, J. C., \& Lazarus, A. J. 2006, GeoRL, 33,9101

Heppner, J. P., Sugiura, M., Skillman, T. L., et al. 1967, JGR, 72, 5417

Hoppe, M. M., Russell, C. T., Eastman, T. E., \& Frank, L. A. 1982, JGR, 87, 643650

Kasper, J. C., Lazarus, A. J., \& Gary, J. P. 2002, GeoRL, 29, 1839

Marsch, E., Ao, X. Z., \& Tu, C. Y. 2004, JGR, 109, A04102

Maruca, B. A., Kasper, J. C., \& Gary, S. P. 2011, PRL, 107, 201101

Osman, K. T., Matthaeus, W. H., \& Hnat, B. 2012, PRL, 108, 261103

Paschmann, G., \& Daly, P. W. 1998, ISSI Scientific Reports Series, 1

Quest, K. B., \& Shapiro, V. D. 1996, JGR, 101, 457

Réme, H., Bosqued, J. M., Sauvaud, J. A., et al. 1997, SSRv, 79, 303

Rosin, M. S., Schekochihin, A. A., Rinson, F., \& Cowley, S. C. 2011, MNRAS, 413, 7

Russell, C. T., Childers, D. D., \& Coleman, P. 1971, JGR, 76, 845

Schlickeiser, R., \& Miller, J. A. 1998, ApJ, 492, 352

Scholer, M., Kucharek, H., \& Shinohara, I. 2003, JGR, 108, 1273

Sentman, D. D., Edmiston, J. P., \& Frank, L. 1981, JGR, 86, 7487

Sentman, D. D., Hoppe, M. M., Thomsen, M. F., Gary, S. P., \& Feldman, W. C. 1983, JGR, 88, 2048

Tu, C. Y., Marsch, E., \& Thieme, K. M. 1989, JGR, 94, 11739

Wilson, L. B., III, Cattell, C. A., Kellogg, P. J., et al. 2009, JGR, 114, A10106 